\title{
Bulk ac conductivity studies of lithium substituted layered sodium trititanates $\left(\mathrm{Na}_{2} \mathrm{Ti}_{3} \mathrm{O}_{7}\right)$
}

\author{
Dharmendra Pal • R. K. Pal · J. L. Pandey • \\ S. H. Abdi $\cdot$ A. K. Agnihotri
}

Abstract Lithium mixed sodium trititanates with 0.3 , 0.5 and $1.0 \mathrm{M}$ percentage of $\mathrm{Li}_{2} \mathrm{CO}_{3}$ (general formula $\mathrm{Na}_{2-X} \mathrm{Li}_{X} \mathrm{Ti}_{3} \mathrm{O}_{7}$ ) have prepared by a high temperature solid-state reaction route. EPR analysis, high temperature range (473-773 K) and variable frequency range $(100 \mathrm{~Hz}-1$ $\mathrm{MHz})$ ac conductivity measurements were carried out on prepared sample. The lithium ions are accommodated with the sodium ions in the interlayer space. The EPR specta of lithium mixed sodium Trititanates confirm the partial reduction of $\mathrm{Ti}^{4+}$ ions to $\mathrm{Ti}^{3+}$. Four distinct regions have identified in the $\operatorname{Ln} \sigma T$ versus $1,000 / T$ plots. Various conduction mechanisms which dependence on concentration, frequency and temperature are reported in this paper for lithium mixed layered sodium Trititanates. The dilation of interlayer space has further been proposed to occur due to inclusion of lithium ions in the interlayer space. The conductivity increases as the concentration of lithium increases. The increase of ionic conductivity in these compounds is due to accommodation of lithium ions with sodium ions in interlayer space.

\section{Introduction}

A family of alkali metal titanates, represented by the general formula $\mathrm{A}_{2} \mathrm{O} . \mathrm{nTiO}{ }_{2}$ or $\mathrm{A}_{2} \mathrm{Ti}_{\mathrm{n}} \mathrm{O}_{2 \mathrm{n}+1}(\mathrm{~A}=$ alkali metal and $2 \leq \mathrm{n} \leq 9$ ) is well known to have characterized layered or tunnel type structures depending upon $n$ values [1-5]. These titanates exhibit diverse potential application associated with their high ion exchange ability. Thus, they are used for the host materials to intermediate organic guest molecules [6-8], to fabricate porous materials $[9,10]$ and to fix the radioactive metal ions [11, 12]. Several non alkali metal members are also prepared by ion exchange reactions [13]. Proton exchanged members can be easily exfoliated into single layers by the intercalation of bulky organic amines to fabricate plate-like particles of titania and thin films of protonic titanates [14]. Other properties of interest for $\mathrm{A}_{2} \mathrm{Ti}_{\mathrm{n}} \mathrm{O}_{2 \mathrm{n}+1}$ titanates includes photo-luminescence and photo catalytic activities [15-17]. The structural transformation behavior of $\mathrm{Na}_{2} \mathrm{Ti}_{3} \mathrm{O}_{7}$ by hydrolysis was investigated in the mild and strong acidic aqueous medium [18]. The crystal structure of $\mathrm{Na}_{2} \mathrm{Ti}_{3} \mathrm{O}_{7}$ consists of layers of composition $\mathrm{Ti}_{3} \mathrm{O}_{7}{ }^{2-}$, like two dimensional sheet centered at the plate (100) plane. The layers are held together by sodium ions, which exist in two different kinds of crystallographic sites. The $\mathrm{Na}_{2} \mathrm{Ti}_{3} \mathrm{O}_{7}$ crystals are monoclinic with unit cell dimensions $a=0.9133 \mathrm{~nm}, b=0.3806 \mathrm{~nm}$, $c=0.8566 \mathrm{~nm}$ and $\beta=101.57^{\circ}$ space group $=\mathrm{P}_{21} / \mathrm{m}$ [4]. O. V. Yakubovich and V. V. Kireev have studied the refinement of this compound [19]. Machida et al. [20] have reported pillaring and photo catalytic properties of partially substituted layered titanates $\mathrm{Na}_{2} \mathrm{Ti}_{3-X} \mathrm{M}_{X} \mathrm{O}_{7}$ and $\mathrm{K}_{2} \mathrm{Ti}_{4-X}$ $\mathrm{M}_{X} \mathrm{O}_{9}(\mathrm{M}=\mathrm{Mn}, \mathrm{Fe}, \mathrm{Co}, \mathrm{Ni}, \mathrm{Cu})$. The layered $\mathrm{Na}_{2} \mathrm{Ti}_{3} \mathrm{O}_{7}$ powders of varying grain sizes have been prepared by solid-state reaction of $\mathrm{Na}_{2} \mathrm{CO}_{3}$ and $\mathrm{TiO}_{2}$ with average particle sizes [21]. The zigzag layers of $\mathrm{Na}_{2} \mathrm{Ti}_{3} \mathrm{O}_{7}$ have 
three dipole moments of 5.0, 5.8 and 6.2D (Debye) [22]. EPR and electrical studies in layered $\mathrm{Na}_{1.9} \mathrm{Li}_{0.1} \mathrm{Ti}_{3} \mathrm{O}_{7}$ and its copper doped derivatives has been reported in my previous research work [23]. EPR and mixed electronic-ionic conductivity studies of pure and manganese doped Layered Potassium lithium Tetra Titanates $\left(\mathrm{K}_{1.9} \mathrm{Li}_{0.1} \mathrm{Ti}_{4} \mathrm{O}_{9}\right)$ have reported in my previous research work [24]. $\mathrm{K}_{2} \mathrm{Ti}_{4} \mathrm{O}_{9}$ has layered lattice of titanium oxygen octahedral, in which the potassium atom occupies the interlayer regions [25]. It also has monoclinic crystal lattice similar to $\mathrm{Na}_{2} \mathrm{Ti}_{3} \mathrm{O}_{7}$ with unit cell dimensions as $a=1.8255 \mathrm{~nm}, b=0.3790 \mathrm{~nm}, c=$ $1.2017 \mathrm{~nm}$ and $\beta=106.43^{\circ}$. The ac conductivity studies in polycrystalline samples of $\mathrm{Na}_{2} \mathrm{Ti}_{3} \mathrm{O}_{7}$ and $\mathrm{K}_{2} \mathrm{Ti}_{4} \mathrm{O}_{9}$ and their $\mathrm{Nb}$ containing product were first reported by Kikkawa et al. [26]. The ion exchange reactions involving polycrystalline samples of $\mathrm{Na}_{2} \mathrm{Ti}_{3} \mathrm{O}_{7}$ and $\mathrm{K}_{2} \mathrm{Ti}_{4} \mathrm{O}_{9}$ are quite well known $[27,28]$. The crystal structure of $\mathrm{Na}_{2} \mathrm{Ti}_{3} \mathrm{O}_{7}$ and $\mathrm{K}_{2} \mathrm{Ti}_{4} \mathrm{O}_{9}$ do suggest possibility of ionic conduction along the layers and thus application of these materials as ion exchanger [28]. The work of Kikkawa et al. [26] has opened the new route to study about the various electrical conduction mechanisms involved in mixed compounds of alkali titanates. In this paper we have reported the EPR and mixed electronicionic ac conductivity studies of mixed layered sodium Trititanates with lithium (molar percentage 0.3, 0.5 and 1.0). The nomenclature for different samples obtained is given in Table 1.

\section{Experimental procedures}

The lithium substituted $\mathrm{Na}_{2} \mathrm{Ti}_{3} \mathrm{O}_{7}$ ceramics samples are prepared through solid state reaction route. To prepare lithium mixed sample of $\mathrm{Na}_{2} \mathrm{Ti}_{3} \mathrm{O}_{7}$ the desired molar percentage of $\mathrm{Li}_{2} \mathrm{CO}_{3}$ (Merck Germany $99.9 \%$ pure) $(0.3,0.5$ and 1.0) were added to the starting mixture of $\mathrm{Na}_{2} \mathrm{CO}_{3}$ and $\mathrm{TiO}_{2}$ powder. The remaining method was same as that of pure $\mathrm{Na}_{2} \mathrm{Ti}_{3} \mathrm{O}_{7}$ [23]. The EPR spectra were recorded on a Varian E-line Century Series spectrometer E-109, which operates at X-band frequencies $(-9.3 \mathrm{GHz})$. All the spectra were recorded with a $100 \mathrm{KHz}$ field modulation. The magnetic field was calibrated accurately with a Varian E-500 digital nuclear magnetic resonance gauss meter.

For ac conductivity measurements the flat faces of the sintered pellets were painted with an air-dried high purity

Table 1 Nomenclatures of the prepared samples

\begin{tabular}{lll}
\hline Samples & Molar percentage & Name \\
\hline $\mathrm{Na}_{1.7} \mathrm{Li}_{0.3} \mathrm{Ti}_{3} \mathrm{O}_{7}$ & 0.3 & SLT-1 \\
$\mathrm{Na}_{1.5} \mathrm{Li}_{0.5} \mathrm{Ti}_{3} \mathrm{O}_{7}$ & 0.5 & SLT-2 \\
$\mathrm{NaLiTi}_{3} \mathrm{O}_{7}$ & 1.0 & SLT-3 \\
\hline
\end{tabular}

silver paste and then mounted in the sample holder evacuated up to $10^{-3}$ mbar for the electrical measurements. The loss tangent $(\tan \delta)$ and parallel capacitance $\left(C_{\mathrm{p}}\right)$ of the palletized samples were directly measured as a function of temperature and frequency by the HP 4194A impedance analyzer. The bulk ac conductivity $(\sigma)$ of the samples were calculated by using expressions-

Conductance $G=\omega C_{\mathrm{p}} \tan \delta$; where $\omega=2 \pi f$

Conductivity $\sigma=G(t / A)$

Where $f$ is the frequency of ac signal, $t$ is the thickness and $A$ is the area of cross-section of the pellet.

\section{Results and discussion}

The EPR spectra of $0.1 \mathrm{M}$ percentage of lithium in $\mathrm{Na}_{2} \mathrm{Ti}_{3} \mathrm{O}_{7}$ is already reported by Pal et al. [29]. The EPR spectra of $\mathrm{Li}$ substituted $\mathrm{Na}_{2} \mathrm{Ti}_{3} \mathrm{O}_{7}\left[\mathrm{Na}_{2-X} \mathrm{Li}_{X} \mathrm{Ti}_{3} \mathrm{O}_{7}\right.$ with $X=0.3,0.5$ and 1.0] shown in Fig. 1. The EPR spectra of Li substituted $\mathrm{Na}_{2} \mathrm{Ti}_{3} \mathrm{O}_{7}\left[\mathrm{Na}_{2-} \mathrm{Li}_{X} \mathrm{Ti}_{3} \mathrm{O}_{7}\right.$ with $X=0.3,0.5$ and 1.0] confirm the presence of $\mathrm{Ti}^{3+}$ ions, which result by the reduction of $\mathrm{Ti}^{4+}$ ions upon lithium. The $\mathrm{g}$ factor is 2.679 and falls in the range characteristic of $\mathrm{d}^{1}$ ions in the distorted octahedral site. The absence of resolved hyperfine structure expected for the two titanium isotopes Ti $(7.75 \%$ abundant, $I=5 / 2)$ and $\mathrm{Ti}(5.5 \%$ abundant, $I=7 / 2)$ with the nuclear magnetic moment suggests that the unpaired spin is shared by several $\mathrm{Ti}$ nuclei. The substitution of lithium ions in the interlayer space does not affect the crystal structure of $\mathrm{Na}_{2} \mathrm{Ti}_{3} \mathrm{O}_{7}$. This confirm the presence of small lithium ions with large sodium ions the interlayer space [29, 30]. With the knowledge of about occupancy of lithium ions in $\mathrm{Na}_{2} \mathrm{Ti}_{3} \mathrm{O}_{7}$ lattice, the ac conductivity results can be discussed region wise.

\subsection{Region I (lowest temperature region)}

Figure 2a, b, c show the ac conductivity curves for SLT-1, SLT-2, SLT-3, respectively. The lowest temperature region exists up to $548,573,623 \mathrm{~K}$, for SLT-1, SLT-2, SLT-3. It is observed that the value of dc conductivity [23] is lower than ac conductivity. This behavior can be explained by the expression $\sigma(\omega)=A \omega^{s}$, where $s$ is less than unity and parameter, $A$ shows little dependence on temperature [31]. The loosened electrons from the negative ions and also from impurities in the crystal may be transported through the solids and would contribute to the conduction. It has been found that a small number of electrons (which are tightly bond to an ion in an ionic lattice) do contribute to weak conduction in some ionic insulators. These electrons are mainly localized closed to an ions (which are tightly 


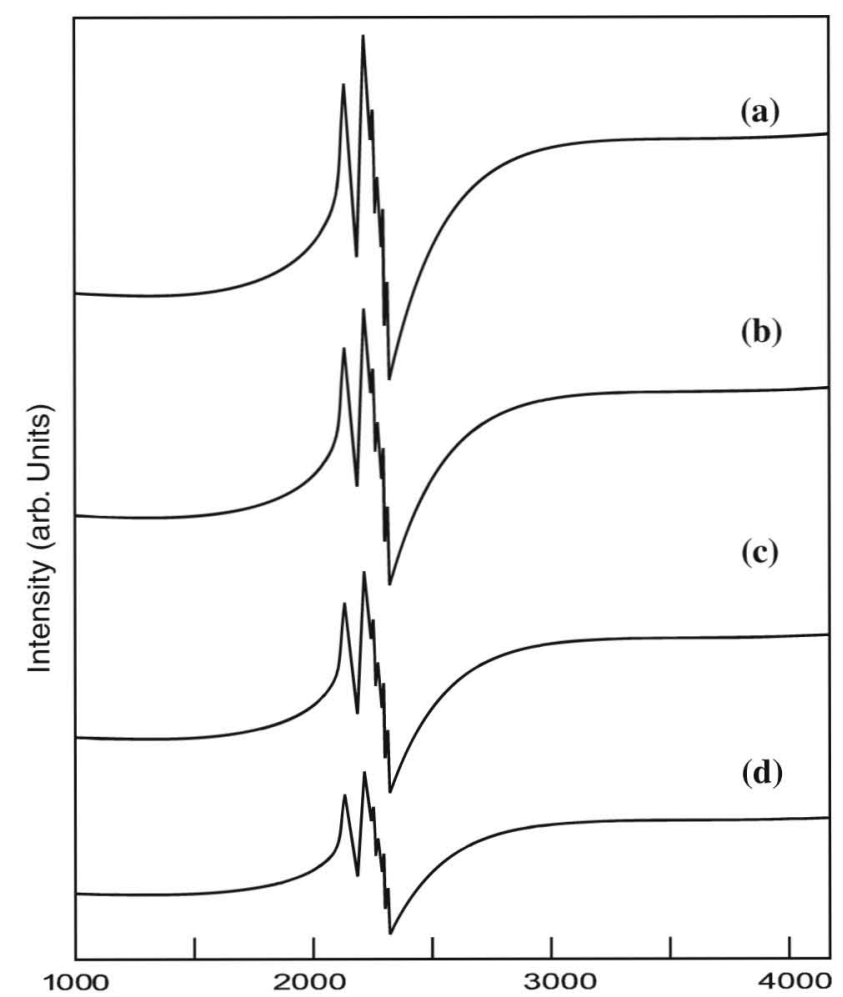

Fig. 1 EPR spectra of lithium mixed sodium trititanates $\mathrm{Na}_{2-X} \mathrm{Li}_{X}$ $\mathrm{Ti}_{3} \mathrm{O}_{7}[X=0.1$ (a) (35), 0.3(b), 0.5(c) and 1.0(d) $]$

bond to an ion in an ionic mainly localized closed an ion). The motion of electrons is so slow that lattice polarization is induced around the electrons. In such a case the conduction mechanism through the crystal can not be explained in terms of usual band theory. The electron flow through the crystal here is connected with its hopping motion from ion (A) to neighbouring ion (B), rather than translational motion in a periodic lattice The polaron is an electron that always moves around, together with the associated lattice-polarization that minimizes its energy. The electronic problem is replaced by the polaronic problem, and the remaining polaron-phonon interactions are assumed to be small. The polaron clearly has lower energy than electron alone, but it has a larger effective mass, since it must carry its lattice distortion with it as it moves the conduction in mixed sodium trititanates is predominantly ionic occurs through the ions in the interlayer space. However, electronic contribution to conduction is quite probable and could occur through $\mathrm{Ti}-\mathrm{Ti}$ chains. The nature of ac conductivity can be interpreted by proposing that the electronic hopping conduction, in which the hopping of electrons through shallow barrier along $\mathrm{Ti}-\mathrm{Ti}$ chain take part in conduction, plays a major roles in this region. Such a frequency dependence of conductivity has been attributed to a wide distribution of relaxation times due to barrier height [32]. Accordingly, the conduction in this region is electronic hopping conduction or polaronic conduction.

\subsection{Region II (anomalous region)}

The anomalous region exists from 548-598 K, 523-573 K, 498-548 K for SLT-1, SLT-2, SLT-3, respectively. The appearance of minor peak at $573 \mathrm{~K}$ for SLT-1 becomes pronounced for SLT-2 and SLT-3. In this anomalous region the initial lower activation energy in the ac conductivity plots may be attributed to dissociation of one of the two cation vacancies associated with the $\mathrm{Li}^{+}$ions occupying the interlayer space and the latter sharp fall in conductivity region to the detachment of even the second cation vacancy from the association of impurity ions. The dissociation of vacancy is quite expected on heating the sample. In EPR spectra this process would be reflected as observance of a band width less anisotropic $g$-value. This is in conformity with our EPR results. The detached cation vacancies could leave the interlayer space and ultimately reach at the intragranular boundary. The cations in interlayer space with one or more cation vacancies removed would pull the opposite $\mathrm{Ti}-\mathrm{Ti}$ layer quite strongly, thus reducing the space between them and hindering the interlayer conduction. In SL:T-2 and SLT-3 the large fall in conductivity in such anomalous region is perhaps due to the fact that sufficient $\mathrm{Li}^{+}$ions occupies the interlayer space. Thus the ionic conduction in anomalous region may be called as 'Hindered interlayer ionic conduction'.

\subsection{Region III (less frequency dependent and most temperature dependent region)}

This entire region shows less frequency dependence and most temperature dependence. The less frequency dependent and most temperature dependent region exist from 598-648 K, 623-698 K, 648-698 K, for SLT-1, SLT-2, SLT-3, The mechanism of conduction in this region is associated interlayer ionic conduction. The total conductivity $\sigma(\omega)$ is expressed [33] by

$\sigma(\omega)=\sigma^{\prime}(\omega)+\sigma^{\prime \prime}(\omega)$.

The mechanism responsible for $\sigma^{\prime}(\omega)$ almost gives a linear dependence of conductivity on frequency as observed in the present work. The mechanism responsible for $\sigma^{\prime \prime}(\omega)$ gives a dielectric loss peak with the distribution of relaxation time.

Accordingly, the conduction in this region is associated interlayer ionic conduction with a part of electronic hopping conduction towards the lower temperature end of this region. 
Fig. 2 ac conductivity plots between $\operatorname{Ln} \sigma T\left(\Omega^{-1} \mathrm{~m}^{-1} \mathrm{~K}\right)$ versus $1,000 / \mathrm{T}$ for a SLT-1, b SLT-2 and c SLT-3
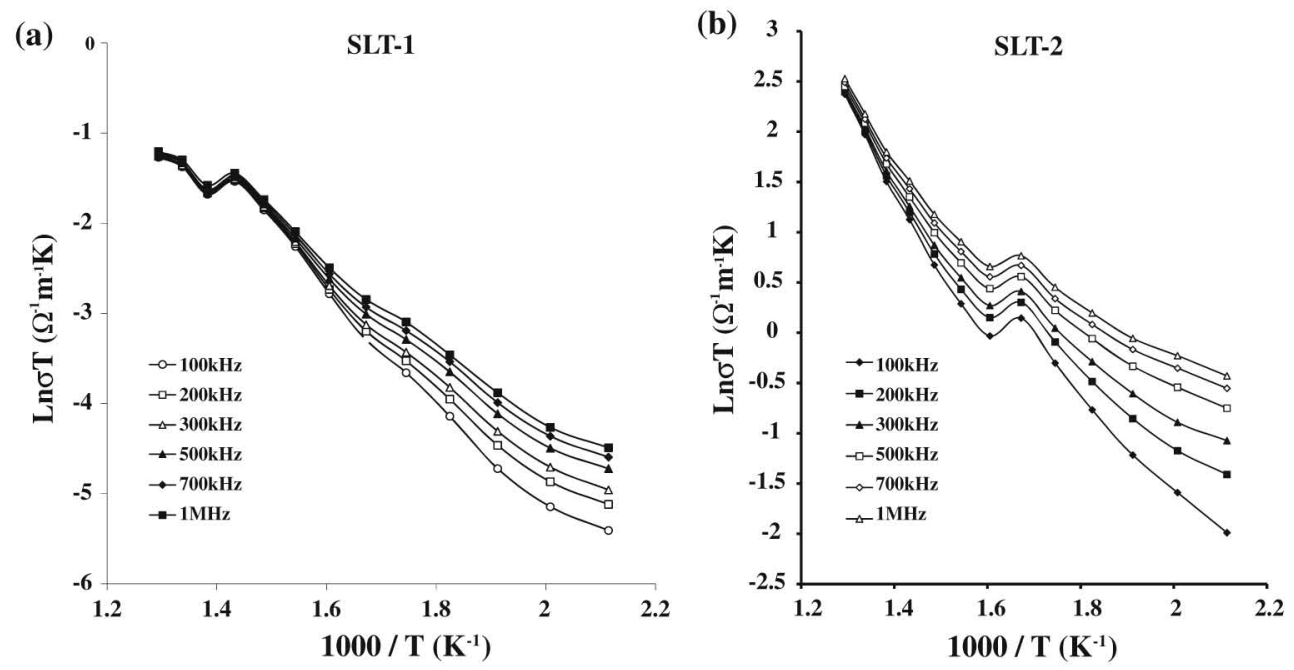

(c)

SLT-3

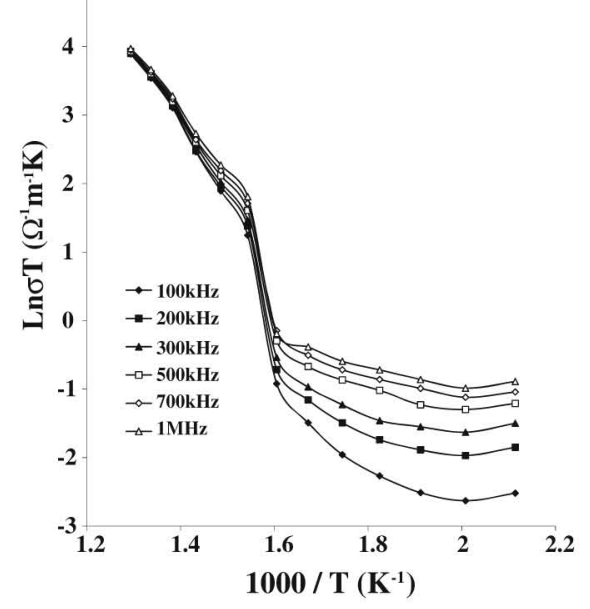

3.4 Region IV (the almost temperature dependent region)

Above 648, 698, $698 \mathrm{~K}$, the almost temperature dependent region exists for SLT-1, SLT-2, SLT-3. In this region the ac conductivity is temperature dependent and frequency independent. The ionic conduction in this region involves unassociated vacancies in the interlayer space. The conduction mechanism in this region can be explained using the expression of Eq. 3. Probably in this region the contribution of $\sigma^{\prime \prime}(\omega)$ starts to dominate. However, $I-V$ dipoles present in the interlayer space has broken up and the mechanism of conduction can now be proposed as unassociated interlayer ionic conduction. The dependence of conductivity on frequency decreases with the increase in temperature for all the compositions. This may be due to electronic hopping conduction is dominant at lower temperature and diminishes with the rise in temperature.

Figure 3 shows the variation of $\operatorname{Ln} \sigma T$ versus composition $(X)$. The conductivity increases as the lithium concentration

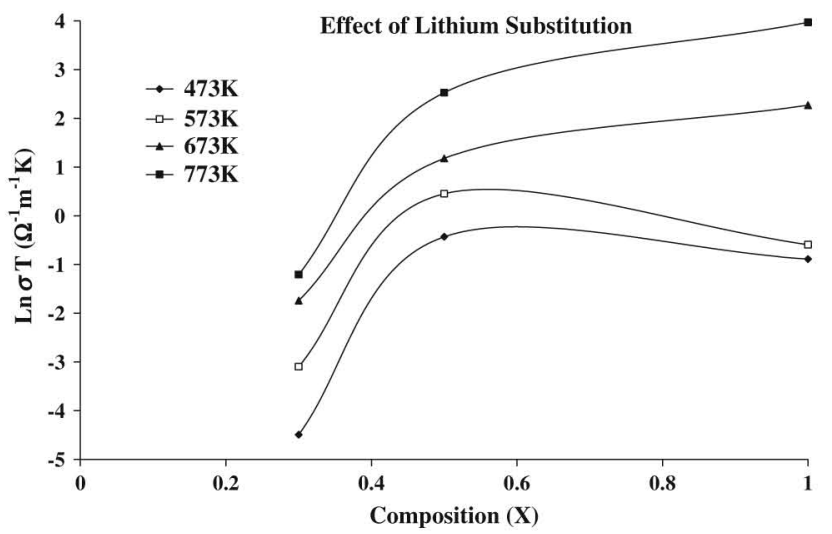

Fig. 3 ac conductivity plots between $\operatorname{Ln} \sigma T\left(\Omega^{-1} \mathrm{~m}^{-1} \mathrm{~K}\right)$ versus composition $(X)$

increases. The electrical conductivity increases as the lithium concentration increases in all layered titanates, this is due to increase of ionic conduction in interlayer space. 


\section{Conclusions}

The outcomes of the present investigations may be summarized as: For the first time-layered polycrystalline $\mathrm{Na}_{2-X} \mathrm{Li}_{X} \mathrm{Ti}_{3} \mathrm{O}_{7}(X=0.3,0.5$ and 1.0) titanates have been synthesized and characterized through EPR and ac conductivity studies. The EPR spectra of lithium mixed sodium Trititanates confirm the partial reduction of $\mathrm{Ti}^{4+}$ ions to $\mathrm{Ti}^{3+}$. ac electrical conductivity studies show that the electronic hopping conduction exists in the entire temperature range of study and diminishes with rise in temperature. In region I the proposed conduction mechanism is electronic hopping conduction or polaronic conduction. The ionic conduction as 'Hindered interlayer ionic conduction' is proposed in region II. The conduction mechanism in region III is associated interlayer ionic conduction with a part of electronic hopping conduction towards the lower temperature end of this region. The unassociated interlayer ionic conduction exists in region IV. The dilation of zigzag interlayer space of $\mathrm{Na}_{2} \mathrm{Ti}_{3} \mathrm{O}_{7}$ at the inclusion of lithium ions has been identified in this work. Layered $\mathrm{Na}_{2-X} \mathrm{Li}_{X} \mathrm{Ti}_{3} \mathrm{O}_{7}(X=0.3,0.5$ and 1.0) ceramics can be put in the class of mixed ionic-electronic materials.

\section{References}

1. S. Anderson, A.D. Wadsley, Nature 107, 499 (1960)

2. K.L. Berry, V.D. Aftandilian, W.W. Gilbert, H.P. Meibohm, H.S. Young, J. Inorg. Nucl. Chem. 14, 231 (1960)

3. S. Anderson, A.D. Wadsley, Acta Chem. Scand. 15, 663 (1961)

4. S. Anderson, A.D. Wadsley, Acta. Cryst. 14, 1245 (1961)

5. S. Anderson, A.D. Wadsley, Acta. Cryst. 15, 194 (1962)

6. H. Izawa, S. Kikkawa, M. Koizumi, J. Solid State Chem. 69, 336 (1987)
7. T. Nakato, K. Kusunoki, K. Yoshizawa, K. Kuroda, M. Kaneko, J. Phys. Chem. 99, 17896 (1995)

8. T. Sasaki, F. Izumi, M. Watanabe, Chem. Mater. 8, 777 (1996)

9. S. Cheng, T.C. Wang, Inorg. Chem. 28, 1283 (1989)

10. J. Kando, N. Shibata, S. Ebina, Y. Ebina, K. Domen, A. Tanaka, J. Phys. Chem. 99, 16043 (1995)

11. T. Sasaki, Y. Komatsu, Y. Fujiki, Chem. Lett. 987, (1981)

12. E.A. Behrens, P. Sylvester, A Clearfield Environ. Sci. Technol. 32, 101 (1998)

13. M. Dion, Y. Piffard, M. Tournoux, J. Inorg. Nucl. Chem. 40, 917 (1978)

14. T. Sasaki, M. Watanabe, H. Hashizume, H. Yamada, H. Nakazawa, J. Am. Chem. Soc. 118, 8329 (1996)

15. Y.I. Kim, S.J. Atherton, E.S. Brigham, T.E. Mallouk, J. Phys. Chem. 97, 11802 (1993)

16. A. Kudo, T. Sakata, J. Mater. Chem. 3, 1081 (1993)

17. A. Kudo, T. Kondo, J. Mater. Chem. 7, 777 (1997)

18. S. Soolee, S.-H. Byeon, Bull. KCS 25(7), 1051 (2004)

19. O.V. Yakubovich, V.V. Kireev, J. Crystallographic Rep. 48(1), 24 (2003)

20. M. Machida, W.M. Xu, H. Taniguchi, J. Yabunaka, T. Kijima, J. Mol. Catal. A Chem. 155, 131 (2004)

21. J. Yang, D. Li, X. Wang, X. Yang, L. Lu, J. Mater. Sc. 38(13), 2907 (2003)

22. S. Ogura, K. Soto, Y. Inoue, Phys. Chem. Chem. Phys. 2, 2449 (2002)

23. D. Pal, P. Chand, R.P. Tandon, Shripal, JKCS 49(6), 560 (2005)

24. D. Pal, Shripal, J. Mater. Sci: Mater. In Electronic 18, 401 (2007)

25. A. Verbaere, M. Tournoux, Bull. Soc. Chim. Fr. 4, 1237 (1973)

26. S. Kikkawa, F. Yasuda, M. Koizumi, Mater. Res. Bull. 20, 1221 (1985)

27. H. Izawa, S. Kikkawa, M. Koizumi, J. Phys. Chem. 86, 5023 (1982)

28. Y. Fujiki, F. Izumi, T. Ohsaka, M. Watanable, Yogya Kyo-Kailsh 85, 475 (1977)

29. D. Pal, R.P. Tandon, Shripal, Indian J. Pure Appl. Phys. 44(6), 435 (2006)

30. A. Abragam, B. Bleaney, Electron Paramagnetic Resonance of Tranisition Ions (Clarendon Press, Oxford, 1970), p. 455

31. V. Chepeleva, DAN SSSR 202, 1042 (1972)

32. P.C. Taylor, P.J. Bray, J. Phys. Chem. Solids 33, 43 (1972)

33. A. Mansingh, R.P. Tandon, J.K. Vaid, Phys. Rev. 21(10), 4829 (1980) 